\title{
Effects of Vibration Fatigue on Compression Strength of Corrugated Fiberboard Containers for Packaging of Fruits during Transport
}

\author{
Hyun Mo Jung ${ }^{1}$, Jeong Gil Park ${ }^{2}$ \\ ${ }^{1}$ Dept. of Visual Design, Kyongbuk Science University, Chilgok, Korea, ${ }^{2}$ Dept. of Biosystems Machinery Engineering, \\ Chungnam National University, Daejeon, Korea
}

Received: January $12^{\text {th }}, 2012$; Revised: February $13^{\text {th }}, 2012$; Accepted: February $28^{\text {th }}, 2012$

\section{Abstract}

Purpose: The compression strength of corrugated fiberboard containers used to package agricultural products rapidly decreases owing to various environmental factors encountered during the distribution of unitized products. The main factors affecting compression strength are moisture absorption, long-term top load, and fatigue caused by shock and vibration during transport. This study characterized the durability of corrugated fiberboard containers for packaging fruits and vegetables under simulated transportation conditions. Methods: Compression tests were done after corrugated fiberboard containers containing fruit were vibrated by an electro-dynamic vibration test system using the power spectral density of routes typically traveled to transport fruits and vegetables in South Korea. Results: To predict loss of compression strength owing to vibration fatigue, a multiple nonlinear regression equation $\left(r^{2}=0.9217, R M S E=0.6347\right)$ was developed using three independent variables of initial container compression strength, namely top stacked weight, loading weight, and vibration time. To test the applicability of our model, we compared our experimental results with those obtained during a road test in which peaches were transported in corrugated containers. Conclusions: The comparison revealed a highly significant $(p \leq 0.05)$ relationship between the experimental and road-test results.

Keywords: Compression strength, Corrugated fiberboard container, Transportation, Vibration fatigue

\section{Introduction}

Damage to agricultural products is mostly caused by breakage of the packing container. In this regard, the compression strength of agricultural packaging is critical and is standardized by the Korean industrial standard and other regulations such as American Society of Testing Materials (ASTM) and International Organization for Standardization (ISO). Container compression strength is generally evaluated by ring crush and edgewise compression strength of the corrugating medium and linerboard paper. The compression strength of corrugated containers decreases owing to many factors encountered during distribution after production. With the ongoing expansion of international

\footnotetext{
*Corresponding author: Hyun Mo Jung

Tel: +82-54-979-9558; Fax: +82-54-972-9558

E-mail: hmjung@kbsu.ac.kr
}

agricultural markets, the use of corrugated fiberboard containers is expected to increase. Therefore, it is necessary to improve the durability and compression strength of corrugated containers.

Many studies have indicated that a container's compression strength depends on the material properties as well as container geometry (length, width, height, and their ratios), dynamic loading conditions during handling and transportation (vibration, impaction, compression), and environmental conditions (humidity, temperature). Vibration particularly affects containers lower in a stack (Nada, 1961; Godshall, 1968), and compression strength of containers can decrease by up to $75 \%$ after continuous shock (Singh, 1987). In one study, the average reduction in compression strength of corrugated containers after United Parcel Service overnight service in the United States was found to be $27.3 \%$ (Pratheepthinthong, 1999). 
Burgess et al. (2005) showed that published strength retention factors greatly overestimated failure timesthe time taken for boxes to collapse under a top load. Rouillard and Sek (2000) carried out sinusoidal sweep vibration tests using a hydraulic servo actuator to measure resonance frequency of corrugated fiberboard containers; they found that resonance occurred at about $14.5 \mathrm{~Hz}$ and reported container breakage after about $20 \mathrm{~min}$ of doing a sinusoidal dwell test at $0.7 \mathrm{G}$ acceleration at the resonance frequency. To understand vibration during distribution and assist in pallet design, Timothy and Marshall (1999) performed a sinusoidal sweep vibration test on pallet packaging ( $3 \sim 50 \mathrm{~Hz}$ frequency) and showed that pallet form and load weight affected resonance frequency. Approximately $64 \%$ of the load is supported in the four perpendicular corners of a container, and the remaining 36\% is supported by the side panels (McKee et al., 1963). Hence, compression strength is mainly governed by perpendicular compression strength and material stiffness. The edgewise compression strength method distinguishes the compression strength of containers from that of the corrugated fiberboard sample without much influence of container dimensions (Gartaganis, 1975; Koning and Stern, 1977; Leake, 1988). Urbanik and Frank (2006) quantified the disparity between results obtained from the McKee equation (McKee et al., 1963) in various published data and presented an improved model for single-wall box-compression strength.

In Korea, several studies have attempted to improve the quality of corrugated fiberboards (Kim and Jung, 2002; Park et al., 1994; Park et al., 1995; Kim et al., 2010), focusing mainly on how compression strength diminishes owing to factors such as temperature, humidity, creep in static conditions, and container dimensions. Some studies optimized compression strength by mechanical methods like finite-element analysis (Kim et al., 2004; Park and Kwon, 2002). The objectives of this study were to deter- mine the loss of compression strength (LCS) of corrugated containers used for fruit packaging in response to vibration fatigue under simulated transportation conditions, and we compared the results with those obtained for containers that were actually shipped.

\section{Materials and Methods}

\section{Corrugated fiberboard containers used to package fruits}

All corrugated containers (boxes) used in this study were regular slotted containers (RSC) made with commercial outer linerboard of $\mathrm{KA}^{180}$ (basic weight, $180 \mathrm{~g} / \mathrm{m}^{2}$; ring crush $22.0 \mathrm{~kg}_{\mathrm{f}}$ ), corrugating medium of $\mathrm{K}^{180}$, and inner, middle linerboard of $\mathrm{K}^{180}$ (basic weight, $180 \mathrm{~g} / \mathrm{m}^{2}$; ring crush $20.5 \mathrm{~kg}_{\mathrm{f}}$ ) that are commonly used in Korea to package fruit. Table 1 lists the size and material specifications of these containers. The dimensions of corrugated containers were similar to those used for fruit packaging in South Korea. Two types of single-wall (SW) and double-wall (DW) corrugated fiberboard containers were used as specified by the agricultural production standard applied in South Korea. All containers used in this study were conditioned to maintain equilibrium in a large thermohygrostat for more than $48 \mathrm{~h}$ at $23 \pm 1^{\circ} \mathrm{C}$ and $50 \%$ relative humidity in accordance with ASTM D685 standard.

\section{Random-vibration and compression tests}

Corrugated fiberboard containers were subjected to vibration fatigue. Each experimental container was placed on a vibration table, top-loaded with a variable dead weight (Figure 1), and then subjected to random vibrations for various periods typical of domestic transportation in Korea. Compression tests were then performed.

Random-vibration tests were carried out using the power

Table 1. Types and physical properties of corrugated fiberboard containers tested

\begin{tabular}{cccccc}
$\begin{array}{c}\text { Container } \\
\text { Type }\end{array}$ & Flute & Symbol & Paper combination & $\begin{array}{c}\text { Dimensions } \\
(\mathrm{L} \times \mathrm{W} \times \mathrm{D}, \mathrm{mm})\end{array}$ & $\begin{array}{c}\text { Compression } \\
\text { strength }(\mathrm{kg})\end{array}$ \\
RSC & A-Flute & $\mathrm{SW}_{1}$ & $\mathrm{KA}^{180} / \mathrm{K}^{180} / \mathrm{K}^{180}$ & $550 \times 366 \times 160$ & $398.15 \pm 55.29$ \\
RSC & B-Flute & $\mathrm{SW}_{2}$ & $\mathrm{KA}^{180} / \mathrm{K}^{180} / \mathrm{K}^{180}$ & $550 \times 366 \times 160$ & $315.20 \pm 49.19$ \\
RSC & EB-Flute & $\mathrm{DW}_{1}$ & $\mathrm{KA}^{180} / \mathrm{K}^{180} / \mathrm{K}^{180} / \mathrm{K}^{180} / \mathrm{K}^{180}$ & $550 \times 366 \times 280$ & $596.42 \pm 66.32$ \\
RSC & AB-Flute & $\mathrm{DW}_{2}$ & $\mathrm{KA}^{180} / \mathrm{K}^{180} / \mathrm{K}^{180} / \mathrm{K}^{180} / \mathrm{K}^{180}$ & $550 \times 366 \times 280$ & $754.54 \pm 78.45$ \\
\hline
\end{tabular}

The values are mean of five determinations \pm standard deviation. RSC, regular slotted container; SW and DW, single- and double-wall corrugated fiberboard container, respectively. 


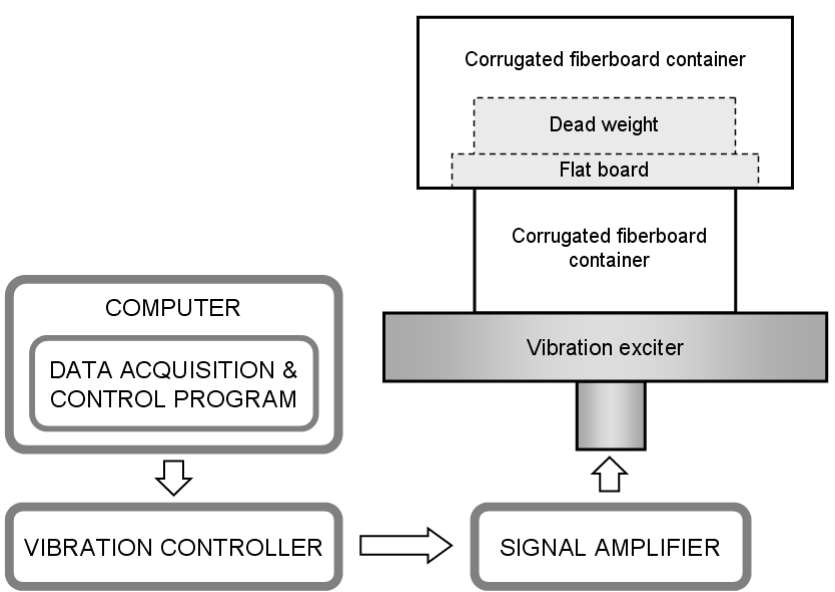

Figure 1. Schematic diagram of the random vibration test apparatus for simulating the real transportation environment of corrugated fiberboard container.

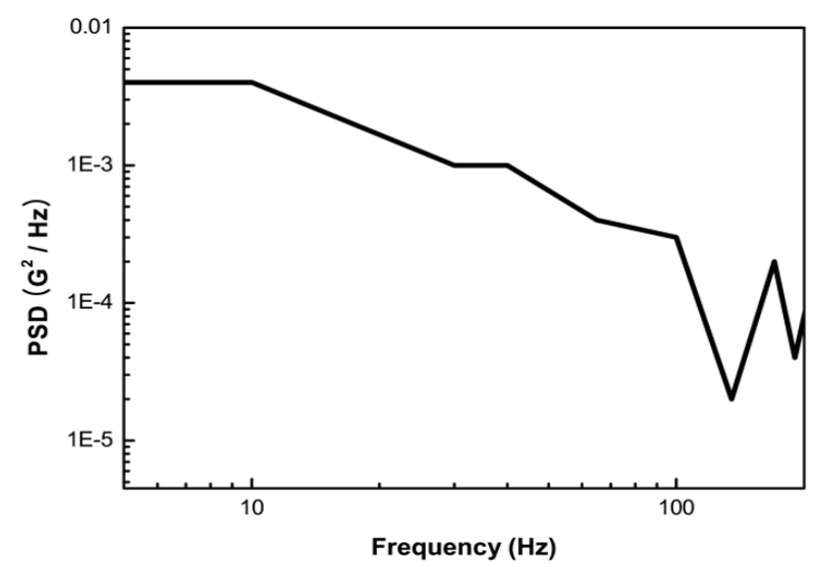

Figure 2. PSD profile of the transportation route for agricultural products from Waeguan to Daejeon in South Korea (Kim, 2007).

spectral density (PSD) profile of domestic transportation routes measured by Kim (2007), which included local highway and express highway routes. Random vibration was set so that the initial input value could incrementally increase starting at least $6 \mathrm{~dB}$ lower than the total level, with random vibrations $(5 \sim 200 \mathrm{~Hz})$ continuing for 185 min maximum, corresponding to the measured transportation time (Choi et al., 2010). Figure 2 shows the PSD profile of a transportation route of packaged fruits from Waeguan to Daejeon in South Korea used to simulate the transportation environment (Kim, 2007).

The compression test had a loading rate of 12.7 $\mathrm{mm} / \mathrm{min}$ in accordance with the ASTM D642 standard. The average container compression strength was based on triplicate measurements of vibration and compression tests. An electro-dynamic vibration actuator (FARMTECH5000, 1 ton, $1 \mathrm{kHz}$, Korea) was used to ensure a stable

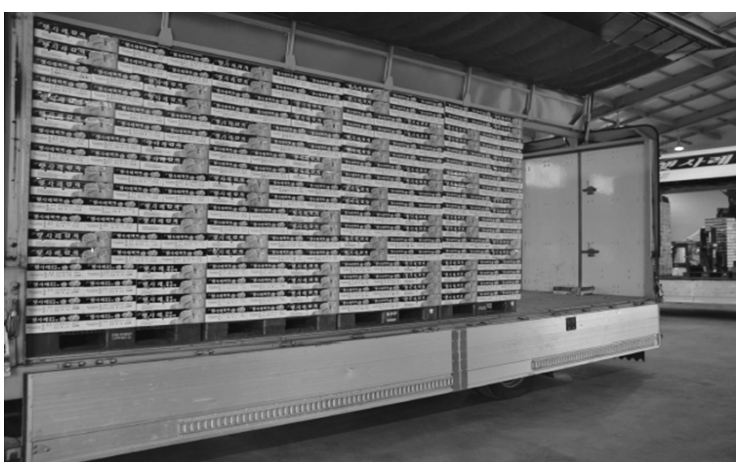

Figure 3. Real transportation test to verify the developed regression model for estimating the loss in compression strength of corrugated fiberboard containers.

acceleration for the vibration experiments. After the random-vibration tests, the same containers were used for compression experiments using a compression tester (DAEYOUNG, 5 ton, Korea).

\section{Verification of the developed regression model}

To verify the regression model for estimating the LCS of corrugated fiberboard containers by vibration fatigue during transport, a transportation test was performed from Daejeon to Seoul. The vehicle used was a 7.5-tonnes truck with air cushion suspensions. Regular slotted containers with EB-flute DW corrugated fiberboard for peach packaging (Figure 3 ) were used as follows: sample container (550 × $366 \times 150 \mathrm{~mm}$ ), a 5-kg packaging unit of a container with peaches, 18 stacked tier, 179 min total transportation time. After the transportation test, 120 stacked containers were randomly selected and peaches were removed prior to performing compression tests. In this study, the container compression strength was estimated without considering other factors such as temperature, relative humidity, and creep in static conditions. The compression tests were performed in the same air conditions as for transportation, and the estimated values of the developed model were compared with measured values in the lab.

\section{Results and Discussion}

\section{Compression strength of corrugated containers decreased with vibration fatigue, load, and transportation time}

To analyze the LCS of corrugated containers with respect 
to initial compression strength, loading weight, and vibration time, simulated transportation and compression tests were performed. Figure 4 represents the forcedeflection curve of a corrugated fiberboard container before the vibration test, when compression strength was maximal. Tables 2 and 3 show the percentage loss of initial compression strength of the corrugated containers from loading weight and vibration time, based on

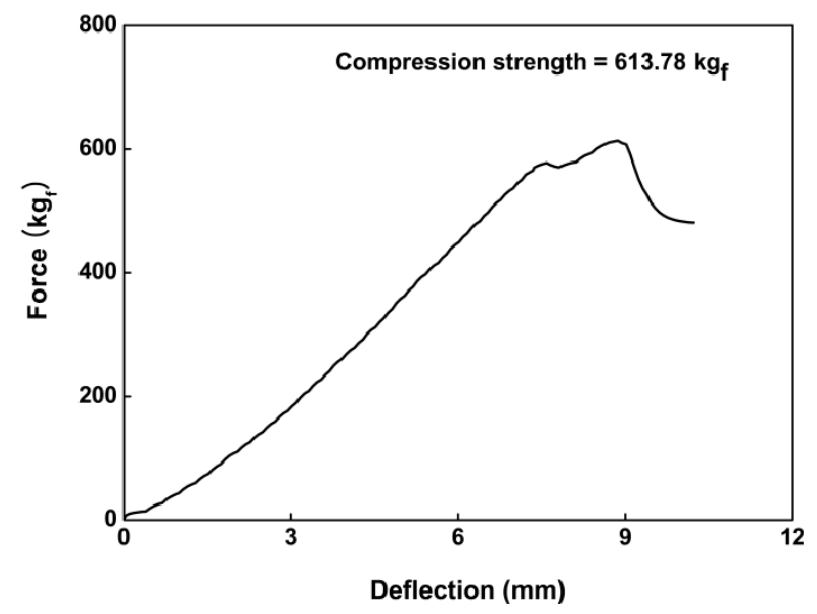

Figure 4. Force-deflection curve of corrugated fiberboard container by compression test. compression strength without any applied vibration. Compression strength decreased with increasing top loading weight and with vibration or transportation time for each sample.

In general, compression strength of each sample decreased with increasing vibration time and loading weight, implying that damage to fruit during transport is directly related to the loss of container strength and breakdown. As container compression strength increased, the LCS decreased in the same transportation environment, indicating that the initial compression strength is directly related to the loss of container strength and stiffness (Jung et al., 2010).

\section{Model for estimating loss of compression strength of corrugated containers caused by vibration fatigue}

To predict the LCS of corrugated containers during transport, a multiple non-linear regression model was developed using independent variables of initial compression strength (ICS) of unprocessed containers, vibration time or transportation time (VT), and loading weight (LW) for random-vibration and compression tests. The correlation analysis of each parameter used to develop the regression model showed that the LW (0.7098) had the highest

Table 2. Percent loss of compression strength of single-wall corrugated fiberboard containers by random-vibration fatigue

\begin{tabular}{|c|c|c|c|c|c|c|c|c|}
\hline \multirow{3}{*}{$\begin{array}{l}\text { Vibration } \\
\text { time } \\
\text { (min) }\end{array}$} & \multicolumn{8}{|c|}{ Loading weight $(\mathrm{kg})$} \\
\hline & \multicolumn{4}{|c|}{$\mathrm{SW}_{1}$} & \multicolumn{4}{|c|}{$\mathrm{SW}_{2}$} \\
\hline & 30 & 70 & 110 & 150 & 30 & 70 & 110 & 150 \\
\hline 40 & 3.2 & 5.5 & 10.3 & 13.7 & 3.5 & 6.8 & 12.9 & 22.1 \\
\hline 80 & 4.7 & 9.1 & 16.6 & 22.1 & 6.3 & 13.6 & 17.7 & 26.6 \\
\hline 120 & 7.5 & 10.3 & 24.3 & 35.7 & 7.1 & 19.4 & 29.3 & 38.3 \\
\hline 160 & 9.1 & 16.9 & 30.1 & 41.3 & 10.3 & 20.3 & 33.6 & 42.9 \\
\hline 200 & 10.3 & 22.1 & 38.6 & 45.4 & 12.7 & 26.1 & 45.4 & 51.5 \\
\hline
\end{tabular}

Values (\%) are averaged. SW, single wall.

Table 3. Percent loss of compression strength of double-wall corrugated fiberboard containers by random-vibration fatigue

\begin{tabular}{|c|c|c|c|c|c|c|c|c|}
\hline \multirow{3}{*}{$\begin{array}{l}\text { Vibration } \\
\text { time } \\
\text { (min) }\end{array}$} & \multicolumn{8}{|c|}{ Loading weight $(\mathrm{kg})$} \\
\hline & \multicolumn{4}{|c|}{$\mathrm{DW}_{1}$} & \multicolumn{4}{|c|}{$\mathrm{DW}_{2}$} \\
\hline & 30 & 70 & 110 & 150 & 30 & 70 & 110 & 150 \\
\hline 40 & 2.3 & 3.1 & 5.5 & 6.3 & 2.1 & 2.8 & 3.5 & 4.5 \\
\hline 80 & 3.1 & 4.3 & 7.4 & 9.5 & 2.4 & 3.1 & 4.4 & 5.3 \\
\hline 120 & 3.5 & 6.2 & 11.3 & 14.1 & 3.6 & 4.7 & 6.8 & 8.9 \\
\hline 160 & 5.1 & 8.1 & 13.7 & 17.5 & 4.6 & 5.5 & 8.6 & 10.6 \\
\hline 200 & 5.3 & 8.2 & 16.9 & 19.5 & 4.8 & 7.1 & 10.2 & 14.4 \\
\hline
\end{tabular}

Values (\%) are averaged. DW, double wall. 
Table 4. Correlation coefficients for comparing the loss of compression strength with initial compression strength, vibration time, and loading weight

\begin{tabular}{ccccc} 
& ICS & VT & LW & LCS \\
\hline ICS & 1.0000 & & & \\
VT & - & 1.0000 & 1.0000 & 1.0000 \\
\hline LW & - & - & 0.7098 & \\
\hline LCS & -0.6891 & 0.6324 & \\
\hline
\end{tabular}

ICS, initial compression strength $\left(\mathrm{kg}_{\mathrm{f}}\right)$; VT, vibration time $(\mathrm{min})$; LW, loading weight $(\mathrm{kg})$; LCS, loss of compression strength (\%).

Table 5. Coefficient of multiple nonlinear regression model for the loss of compression strength ( $95 \%$ level) by vibration fatigue

\begin{tabular}{|c|c|c|c|c|c|}
\hline \multirow[t]{2}{*}{ Variables } & \multicolumn{4}{|c|}{$L C S(L W, V T, I C S)=\frac{L W^{a} \times V T^{b}}{I C S^{c}}$} & \multirow[t]{2}{*}{$r^{2}$} \\
\hline & Coefficient & Standard error & t-ratio & Prob (t) & \\
\hline$a$ & 0.9112 & 0.0547 & 16.6394 & 0.0 & \multirow{3}{*}{0.9217} \\
\hline$b$ & 1.1519 & 0.0611 & 18.8405 & 0.0 & \\
\hline$c$ & 1.1357 & 0.0553 & 20.5562 & 0.0 & \\
\hline
\end{tabular}

LCS, loss of compression strength (\%); ICS, initial compression strength $\left(\mathrm{kg}_{\mathrm{f}}\right)$; VT, vibration time (min); LW, loading weight $(\mathrm{kg})$.

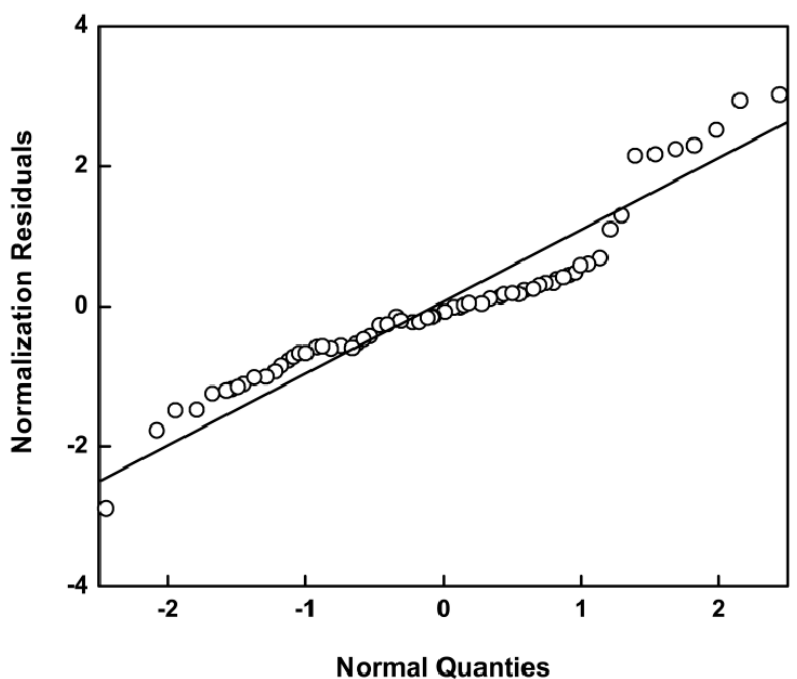

Figure 5. Residual normal probability plot of developed model for estimating the loss of compression strength of corrugated fiberboard containers.

correlation, followed by the VT (0.6324), and ICS (-0.6891) with LCS. All of the parameters showed high correlation (Table 4). Based on correlation analysis, LW had the most influence on LCS based on Korean domestic transportation. Therefore, packaging containers located lower on a pallet experienced excessive LCS after vibration fatigue during transportation. Moreover, container breakage affected the stability of pallet-packaged freight and also caused damage to packaged products.
As shown in Table 5, the coefficient of multiple determination $\left(r^{2}\right)$ of the developed multiple regression model was 0.9217 and the root mean square error (RMSE) was 0.6347 . Analysis of variance of these values showed a high significance $(p \leq 0.05)$ in the developed model using STATISTICA 7.1 for Windows (StatSoft Inc., Tulsa, OK, USA).

Figure 5 shows a normal probability plot of the residuals used to assess the validity of our model. If the error terms follow a normal distribution, they will fall on a straight line on the normal probability plot. Because they are estimates of the error terms, the residuals should exhibit similar properties. Our model is valid because the normal probability plot for the model followed a linear trend.

\section{Verification of the developed regression model}

Figure 6 shows the relationship between the results estimated by the developed model and those measured after a real transportation test of packaged containers of peaches. There were highly significant $\left(r^{2}=0.8962, p \leq\right.$ 0.05 ) relationships in both output values, even though there were some outliers in the output range, which may have been generated by slight movement of containers by shock and vibration during the real transportation experiment. Corrugated containers that did not receive any 
Jung et al. Effects of Vibration Fatigue on Compression Strength of Corrugated Fiberboard Containers for Packaging of Fruits during Transport

Journal of Biosystems Engineering • Vol. 37, No. 1, $2012 \cdot$ www.jbeng.org

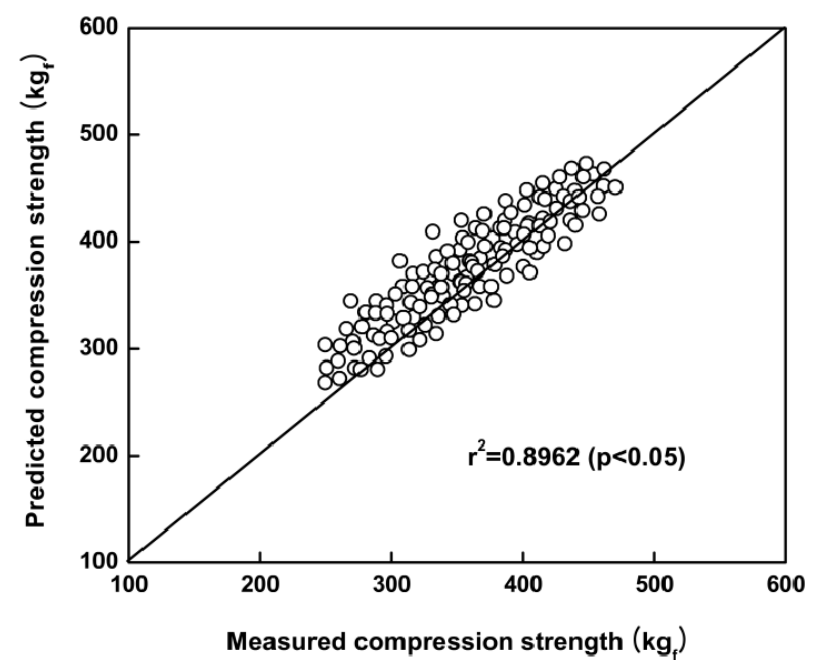

Figure 6. Validation scatter plots in compression strength of corrugated fiberboard containers. Predicted values were obtained by developed model and measured values were obtained by compression tests after real transportation.

vibration fatigue had an average ICS of $491.28 \pm 43.29 \mathrm{~kg}_{\mathrm{f}}$.

\section{Conclusions}

Random-vibration and compression experiments were performed on corrugated fiberboard containers to analyze their LCS by vibration fatigue during transport. The multiple nonlinear regression equation $\left(r^{2}=0.9217, R M S E\right.$ $=0.6347$ ) for predicting the LCS of corrugated fiberboard containers by vibration fatigue was developed using three independent variables: container's ICS, top stacked LW, and VT. Analysis of variance of these values showed a high significance $(p \leq 0.05)$ in the developed regression model. A real transportation test was performed using corrugated containers for peach packaging to verify the developed model, and there was a highly significant $(p \leq$ 0.05 ) relationship between the results estimated by the developed model and those measured after the real transportation test.

\section{Conflict of Interest}

No potential conflict of interest relevant to this article was reported.

\section{References}

ASTM. 2005. D642-00: Standard methods for determining compressive resistance of shipping containers, components, and unit loads. West Conshohocken, PA: ASTM. ASTM. 2007. D685-93: Standard practice for conditioning paper and paper products for testing. West Conshohocken, PA: ASTM.

Burgess, G., S. P. Singh and M. Srinangyam. 2005. Predicting collapse times for corrugated boxes under constant top load using short-term creep tests. Journal of Testing and Evaluation 33(4):284-286.

Choi, S. R., Y. H. Lee, D. S. Choi and M. S. Kim. 2010. Damage at the peach due to vibrational Stress during transportation simulation test. Journal of Biosystems Engineering 35(3):182-188 (In Korean).

Gartaganis, P. A. 1975. Strength properties of corrugated containers. Tappi 58(11):102-108.

Godshell, W. D. 1968. Effects of vertical dynamic loading on corrugated fiberboard containers. Research Paper FPL 94. Madison, WI: Forest Products Laboratory.

Jung, H. M., M. S. Kim and R. Auras. 2010. Effects of packaging materials processed with oak charcoal on the quality of oriental pears during storage and distribution. Journal of Biosystems Engineering 35(5): 316-322 (In Korean).

Kim, M. S. and H. M. Jung. 2002. Vibration characteristics of corrugated fiberboard boxes for packages of pears. Journal of the Korean Society for Agricultural Machinery 27(5):391-398 (In Korean).

Kim, M. S., H. M. Jung and K. B. Kim. 2004. Vibration analysis of pears in packaged freight using finite element method. Journal of the Korean Society for Agricultural Machinery 29(6):501-507 (In Korean).

Kim, G. S. 2007. Vibration behavior of the fruits and the packaged freight and their quality changes at simulated transportation environment. BS Thesis. Daejeon, Korea: Chungnam National University, Department of Biosystems Machinery Engineering.

Kim, G. S., J. M. Park and M. S. Kim. 2010. Functional shock responses of the pear according to the combination of the packaging cushioning materials. Journal of Biosystems Engineering 35(5):323-329 (In Korean).

Koning, J. W. and R. K. Stern. 1977. Long-term creep in corrugated fiberboard containers. Tappi 60(12):128-131.

Leake, C. H. 1988. Measuring corrugated box performance. Tappi Journal Oct.:71-75.

McKEE, R. C., J. W. Gander and J. R. Wachuta. 1963. Compression strength formula for corrugated boxes. Paperboard Packaging (Aug.):144-159. 
Nada, S. 1961. The influence of vibration on the compressive strength and deflection at maximum compression of U.S. and Japanese B-flute corrugated containers. East Lansing, MI: Michigan State University, College of Agriculture.

Park, J. M., S. H. Kwon and M. S. Kim. 1994. Improvement and analysis of stacking durability of corrugated fiberboard boxes for agricultural products - Moisture absorption properties and compressive strength reduction -. Journal of the Korean Society for Agricultural Machinery 19(4):358-368 (In Korean).

Park, J. M., M. S. Kim and S. W. Joung. 1995. Static cushioning properties of corrugated fiberboard and creep behavior of boxes. Journal of the Korean Society for Agricultural Machinery 20(4):323-332 (In Korean).

Park, J. M. and S. W. Kwon. 2002. Finite element analysis of a ventilating box structure. Journal of the Korean Society for Agricultural Machinery 27(6):557-564 (In Korean).
Pratheepthingthong, S. 1999. Loss of compression strength in corrugated boxes during overnight shipments in UPS. MS thesis. East Lansing, MI: Michigan State University.

Rouillard, V. and M. A. Sek. 2000. Monitoring and simulating non-stationary vibrations for package optimization. Packaging Technology and Sciences 13:149-156.

Singh, S. P. 1987. The effect of mechanical shocks on the compressive strength of corrugated containers. East Lansing, MI: Michigan State University, School of Packaging.

Timothy, G. W. and S. W. Marshall. 1999. The effect of pallet connection stiffness, deck stiffness and static load level on the resonant response of pallet decks to vibration frequencies occurring in the distribution environment. Packaging Technology and Science 12(2): 47-55.

Urbanik, T. J. and B. Frank. 2006. Box compression analysis of world-wide data spanning 46 years. Wood and Fiber Science 38(3):399-416. 